\title{
Pulmonary matrix metalloproteinase-9 activity in mechanically ventilated children with respiratory syncytial virus
}

\author{
Michele Y.F. Kong ${ }^{1}$, John P. Clancy ${ }^{2}$, Ning Peng ${ }^{1}$, Yao Li ${ }^{1}$, Tomasz J. Szul ${ }^{3}$, Xin Xu ${ }^{3}$, \\ Robert Oster ${ }^{3}$, Wayne Sullender ${ }^{4}$, Namasivayam Ambalavanan $^{1}$, J. Edwin Blalock ${ }^{3}$ \\ and Amit Gaggar ${ }^{3}$
}

Affiliations: 'Dept of Pediatrics, University of Alabama at Birmingham, Birmingham, AL, ${ }^{2}$ Cincinnati Children's Hospital Medical Center, Cincinnati, $\mathrm{OH},{ }^{3}$ Dept of Medicine, University of Alabama at Birmingham, Birmingham, $\mathrm{AL}$, and ${ }^{4}$ Center for Global Health, Colorado School of Public Health, Aurora, CO, USA.

Correspondence: M.Y.F. Kong, 504 ACC, 1600 7th Avenue South, Children's of Alabama, Birmingham, AL 35233, USA. E-mail: mkongapeds.uab.edu

ABSTRACT Respiratory syncytial virus (RSV) infection is a potent stimulus for airway epithelial expression of matrix metalloproteinase (MMP)-9. MMP-9 activity in vivo is a predictor of disease severity in children with RSV-induced respiratory failure.

Human airway epithelial cells were infected with RSV A2 strain and analysed for MMP-9 and tissue inhibitor of metalloproteinase (TIMP)-1 (a natural inhibitor of MMP-9) release. In addition, endotracheal samples from children with RSV-RF and controls (non-RSV pneumonia and nonlung disease controls) were analysed for MMP-9, TIMP-1, human neutrophil elastase and myeloperoxidase activity.

RSV infection of airway epithelia was sufficient to rapidly induce MMP-9 transcription and protein release. Pulmonary MMP-9 activity peaked at $48 \mathrm{~h}$ in infants with RSV-induced respiratory failure. In the RSV group, MMP-9 activity and MMP-9/TIMP-1 ratio imbalance predicted higher oxygen requirement and worse paediatric risk of mortality scores. The highest levels of human neutrophil elastase and myeloperoxidase activity were measured in the RSV cohort; however, unlike MMP-9, these neutrophil markers failed to predict disease severity.

These results support the hypothesis that RSV is a potent stimulus for MMP-9 expression and release from human airway epithelium, and that MMP-9 is an important biomarker of disease severity in mechanically ventilated children with RSV lung infection.

$@$ ERSpublications

MMP-9 is an important biomarker of disease severity in mechanically ventilated children with RSV lung infection http://ow.ly/tgIj5

Received: June 202013 | Accepted after revision: Nov 082013 | First published online: Dec 052013

Support statement: This study was supported by Pediatric Critical Care Scientist Development Program grants to M. Kong (NICHD 5K12HD047349) and A. Gaggar (HL102371).

Conflict of interest: None declared.

Copyright @ERS 2014 


\section{Introduction}

Respiratory syncytial virus (RSV) infection is a common cause of respiratory disease in infants, and can be particularly severe in infants who are premature, have chronic lung disease, congenital heart disease or are immunocompromised [1,2]. Furthermore, RSV infection is a trigger for acute exacerbations in chronic lung diseases, such as cystic fibrosis (CF) and chronic obstructive pulmonary disease (COPD) [3, 4], and is a risk factor for recurrent wheezing in children post-infection [5]. Despite the substantial morbidity and mortality associated with RSV infection, treatment remains primarily supportive, and antiviral agents have not proved to be an effective therapy for severe RSV disease.

Matrix metalloproteinase (MMP)-9 is one of 25 members of the MMP family, which consists of ubiquitous enzymes that are capable of degrading various extracellular matrix proteins [6]. MMPs are tightly regulated, including binding to peptide inhibitors (such as tissue inhibitors of metalloproteinase (TIMP)-1, -2 and -3), and activation that requires proteolytic cleavage and/or oxidation [7]. MMP-9 is prominently expressed in the lung, and while it is involved in many normal homeostatic processes, dysregulated MMP-9 activity has been described in a number of diseases, including asthma [8, 9], emphysema [10], COPD [11], bronchopulmonary dysplasia [12] and in neutrophil-dominated paediatric lung diseases including CF [13, 14] and acute lung injury (ALI) [15]. Recent evidence also suggests that MMP-9 may be a key regulator of neutrophil recruitment through the generation of chemotactic collagen fragments in vitro and in vivo $[16,17]$.

Previously we have reported high levels of MMP-9 in the respiratory secretions of paediatric patients with ALI caused by a variety of insults, including RSV and other viral infections, and an inverse relationship between MMP-9 activity and ventilator-free days [18]. In the current study we focused on relationships between RSV infection of the airway and MMP-9 activity in vitro and in vivo. RSV was a potent and specific stimulus of MMP-9 expression and release from primary human airway epithelial cells (HAECs) and MMP-9 activity correlated with important clinical outcome measures in RSV-infected infants, including oxygen requirement and paediatric risk of mortality (PRISM) scores.

\begin{tabular}{|c|c|c|c|c|}
\hline & Nonlung disease controls" & Non-RSV pneumonia controls & RSV-induced respiratory failure ${ }^{\pi}$ & p-value \\
\hline Subjects & 46 & 29 & 42 & \\
\hline Age months & $21.6 \pm 4.6$ ( 4 weeks -11 years) & $10.45 \pm 2.9$ (3 weeks-7 years) & $2.8 \pm 0.5$ ( 1 week -5 months) & $<0.001$ \\
\hline Sex & & & & 0.26 \\
\hline $\begin{array}{l}\text { Length of } \\
\text { intubation days }\end{array}$ & $4.9 \pm 0.4$ & $9.2 \pm 1.5$ & $5.3 \pm 0.6$ & 0.006 \\
\hline $\begin{array}{l}\text { Length of PICU } \\
\text { stay days }\end{array}$ & $6.1 \pm 0.4$ & $10.03 \pm 1.5$ & $7.36 \pm 0.9$ & 0.049 \\
\hline PRISM-12 & $4.78 \pm 0.8$ & $8.8 \pm 1.4$ & $5.7 \pm 0.7$ & 0.003 \\
\hline PRISM-24 & $4.47 \pm 0.6$ & $6.8 \pm 0.8$ & $3.3 \pm 0.6$ & 0.004 \\
\hline \multirow{3}{*}{ Diagnosis } & Altered mental status 4 & & & \\
\hline & Muscular weakness 2 & & & \\
\hline & Other ${ }^{+} 8$ & & & \\
\hline
\end{tabular}

Data are presented as $n$, mean \pm SEM (range) or mean \pm SEM, unless otherwise stated. All control subjects who developed new radiographic changes while on the ventilator or acquired a new viral or bacterial infection were excluded from the study. PICU: paediatric intensive care unit; PRISM-12: paediatric risk of mortality in $12 \mathrm{~h}$; PRISM-24: paediatric risk of mortality in $24 \mathrm{~h}$; FlO ${ }_{2}$ : inspiratory oxygen fraction. ${ }^{\#}$ : Nonlung disease controls had no evidence of acute or chronic lung disease, with minimal ventilator requirements (low $\mathrm{FlO}_{2}$, positive end-expiratory pressure, tidal volume and ventilatory ratel and clear chest radiographs; ": having a positive RSV culture on admission to the hospital; ${ }^{+}$: includes subjects with arrhythmia, posterior spinal fusion, cervical spine injuries and nasopharyngeal mass. ${ }^{\S}$ : pneumonia secondary to Staphylococcus aureus (n=9), Pseudomonas aeruginosa $(n=3)$, Streptococcus pneumoniae $(n=3)$ and Serratia marcescens $(n=1) ;{ }^{f}$ : pneumonia secondary to influenza $(n=5)$, adenovirus $(n=3)$ and parvovirus $(\mathrm{n}=1) ;{ }^{\# \#}$ : pneumonia secondary to Candida albicans $(\mathrm{n}=2)$. 


\section{Material and methods}

\section{Enrolment of paediatric intensive care unit patients with respiratory failure}

This observational human study was performed in the paediatric intensive care unit (ICU) at the Children's Hospital of Alabama (Birmingham, AL, USA) with local institutional review board (IRB) approval (protocol X080724010). Informed consent was obtained from the legal guardians of the subjects prior to enrolment. Table 1 provides a summary of demographic and diagnostic information on the paediatric subjects with RSV-induced respiratory failure (RSV-RF) $(\mathrm{n}=42)$ and controls (non-RSV pneumonia, $\mathrm{n}=29$ and nonlung disease controls, $\mathrm{n}=46$ ).

\section{Endotracheal aspirate collection and processing}

Tracheal aspirates were collected from RSV and control subjects at $48 \mathrm{~h}$ of intubation, and at day 5 for those who remained mechanically ventilated beyond the first 2 days. Samples were collected via endotracheal tube suctioning using an 8-French suction cannula (CareFusion, San Diego, CA, USA) and centrifuged at $500 \times g$ for $10 \mathrm{~min}$ to separate cells and mucus (pellet) from supernatant. Separate aliquots were subsequently saved at $4{ }^{\circ} \mathrm{C}$ for further analysis.

\section{RSV infection of airway cell cultures}

All airway cells were obtained from lung transplant recipients following informed and signed consent under an IRB-approved protocol. First or second passage cells were seeded onto permeable supports and grown at an air-liquid interface using previously described methods [19]. The RSV A2 strain was obtained from the American Type Culture Collection (ATCC, Manassas, VA, USA) and cultured in HEp-2 human nasopharyngeal carcinoma cells (CCL-23; ATCC), using a previously described sucrose purification method [20]. HAECs were infected with RSV at several different multiplicities of infection (MOI). RSV titres were determined by plaque assay techniques in HEp2 cells [21] and expressed as plaque forming units $\cdot \mathrm{mm}^{-1}$. For ultraviolet (UV)-inactivation of RSV, aliquots of RSV stocks were inactivated by exposure to $1800 \mathrm{~mJ}$ of radiation in a Stratalinker UV cross-linker (Stratagene, La Jolla, CA, USA). For studies with heat-killed RSV, aliquots of viral stocks were boiled for $45 \mathrm{~min}$ [22].

Initial experiments included the examination of MMP and TIMP expression with RSV infection of HAECs at the 48-h time point. To test the hypothesis that duration of infection also results in increased MMP-9 and TIMP expression, mRNA levels were determined at days 2, 3 and 6 post-infection.

\section{Immunoblots and zymography analysis of MMP-9 from HAECs and endotracheal samples}

Samples were subjected to electrophoresis through $7.5 \%$ sodium dodecyl sulfate polyacrylamide gel electrophoresis gel. The membrane was incubated with primary MMP-9 antibody (MAB 911; R\&D Systems, Minneapolis, MN, USA), followed by incubation with species-specific IgG horseradish peroxidase conjugates (at dilutions of 1:5000) the next day. The immunoblot was then developed using enhanced chemiluminescent kits (GE Healthcare, Piscataway, NJ, USA). Zymography analyses of study samples were performed using previously published techniques [23, 24].

Measurement of MMP-9, TIMP, human neutrophil elastase and myeloperoxidase levels MMP-9 activity was quantified using an established fluorometric assay (\#F9M00; R\&D Systems). TIMP-1 (\#DTM100; R\&D Systems), myeloperoxidase (MPO) (\#475919; Calbiochem, Billerica, MA, USA) and human neutrophil elastase (HNE) (CBA016; Calbiochem) concentrations were measured using established sandwich enzyme immunoassay techniques.

\section{Statistical analysis}

Descriptive statistics were computed for each study variable of interest, including mean, standard error of the mean, median and range. Since the distributions of the data for the tracheal aspirate inflammatory markers (active MMP-9, total MMP-9, TIMP-1, MMP-9/TIMP-1 ratio, HNE and MPO), as well as those for the other continuous variables (days of intubation, days of paediatric ICU stay, oxygen, PRISM in $12 \mathrm{~h}$ (PRISM-12), PRISM-24 and age), deviated from a normal distribution, these data were logarithmically transformed using a $\log _{10}$ scale prior to statistical analysis. The log-transformed variables were determined to follow a normal or approximate normal distribution through the use of stem-and-leaf plots, normal probability plots and the Kolmogorov-Smirnov test. Demographic and diagnostic comparisons between RSV, disease control and control subjects were performed using ANOVA for quantitative variables and the Chi-squared test for sex. Overall comparisons between RSV, disease control and control subjects were performed using analysis of variance, while comparisons including covariates of interest such as age, sex and measures of disease severity (PRISM scores and oxygen concentration) were performed using ANCOVA. The Tukey-Kramer multiple comparisons test was used as the post hoc test of choice. Comparisons between 

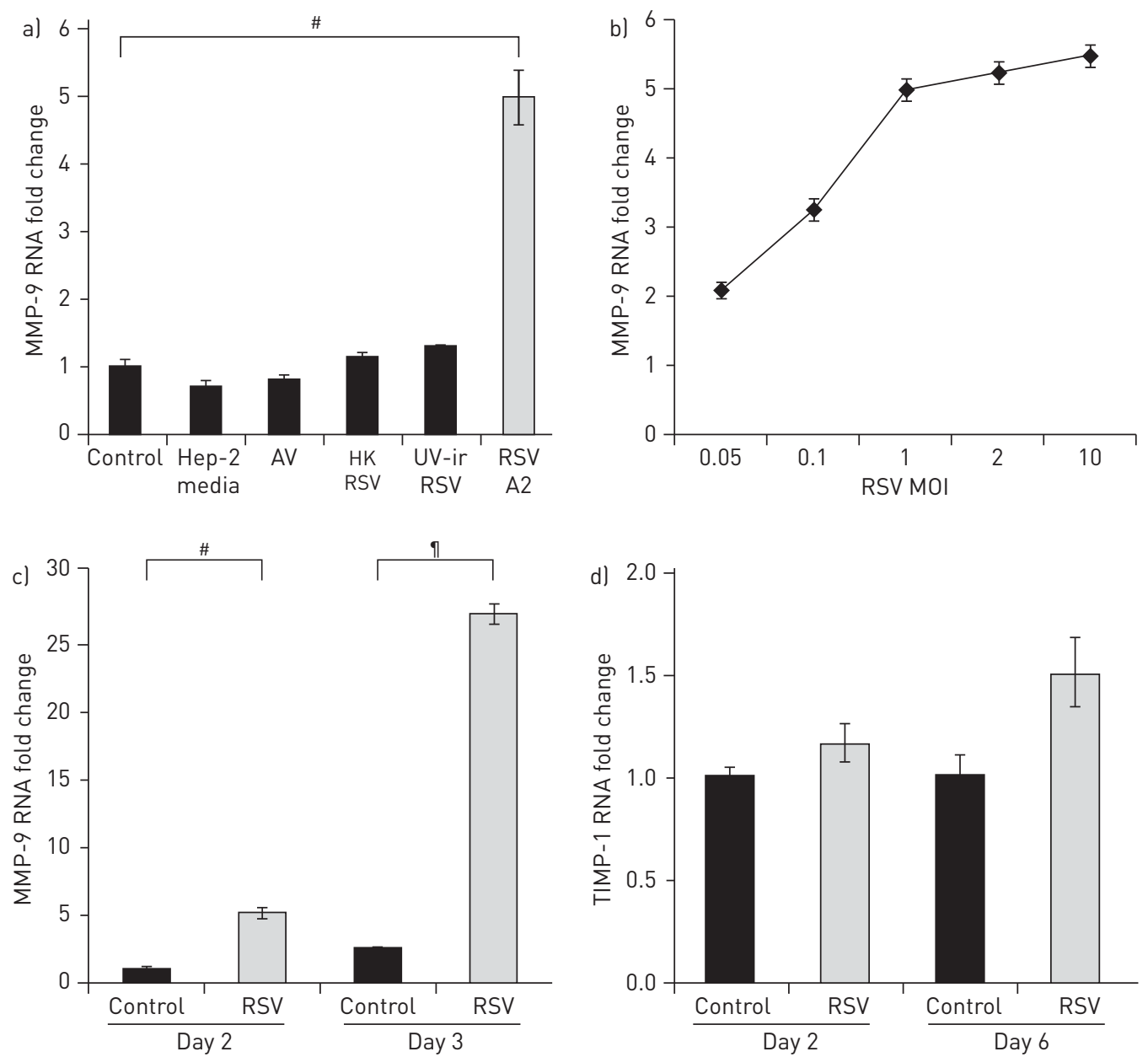

FIGURE 1 Matrix metalloproteinase (MMP)-9 and tissue inhibitor of metalloproteinase (TIMP)-1 mRNA levels compared with controls. Respiratory syncytial virus (RSV) infection of human airway epithelial cells (HAECs) resulted in increased MMP-9 transcription, but had no effect on TIMP-1. a) MMP-9 mRNA level was approximately five-fold higher in HAECs infected with RSV A2 strain (multiplicity of infection (MOI) 0.1) compared to control (mock noninfected) at $48 \mathrm{~h}$ post-infection. Infection of airway epithelia with replicating adenovirus (AV), heat killed (HK) and ultravioletirradiated (UV-ir) RSV resulted in similar levels of MMP-9 transcription when compared with the noninfected cells. Similarly, treatment of cells with Hep-2 media (media used for RSV propagation) had no measureable effects on MMP-9 transcription. b) dose-response effects of RSV infection on MMP-9 transcription. Control was MMP-9 mRNA levels measured after infection with RSV at a MOI of 0.01. At day 2 post-infection, increasing RSV MOI (0.05-10) resulted in exponential increases in MMP-9 transcription. However, MMP-9 mRNA levels reached a plateau at MOI of 1, with similar MMP-9 mRNA levels at MOI of 10 versus 1. c) MMP-9 mRNA levels in RSV-infected cells were five-fold higher than control (mock noninfected) at $48 \mathrm{~h}$ post-infection, and 11-fold higher compared with noninfected controls at day 3 post-infection. Baseline MMP-9 transcription increased with time, as seen in control (mock noninfected) (MMP-9 mRNA was 2.5 -fold higher in control cells at day 3 relative to day $2 ; \mathrm{p}<0.05)$, but the increase in MMP-9 transcription was significantly higher in the RSV-infected cells ( 27 -fold increase relative to control cells on day 2; $<<0.0001$ ). d) TIMP-1 transcription was similar between RSV and control (mock noninfected) airway epithelial cells on days 2 and 6 post-infection. ${ }^{\#}: \mathrm{p}<0.03{ }^{\top}: \mathrm{p}<0.002$.

48-h and day 5 measurements of active MMP-9, total MMP-9, TIMP-1 and MMP-9/TIMP-1 ratio were performed using the Wilcoxon signed-rank test due to the small number of subjects that had available data at both time points. Pearson correlation analysis was used to determine correlations between pairs of quantitative variables and Spearman correlation analysis was used to determine correlations between sex and quantitative variables. Simple and multiple linear regression analyses were used to assess the ability of the inflammatory markers to predict the outcome measures (days of intubation and days of paediatric ICU stay) as well as measures of disease severity. Where possible, nonparametric statistical analyses (including the Kruskal-Wallis test and Spearman correlation analyses) corresponding to the above analyses were performed, and these analyses yielded results that are similar to those obtained by the parametric analyses described earlier. Statistical tests were two-sided and were performed using a 5\% significance level (i.e. $\alpha=0.05$ ). Statistical analyses were performed using SAS software (version 9.3; SAS Institute, Inc., Cary, NC, USA). 


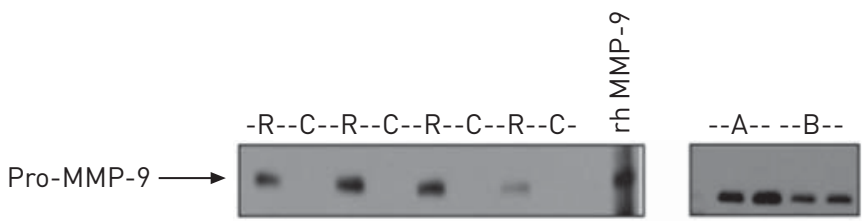

FIGURE 2 Matrix metalloproteinase (MMP)-9 protein release was increased in respiratory syncytial virus (RSV)-infected human airway epithelial cells (HAECs). MMP-9 protein $(92 \mathrm{kDa}$ ) was detected in RSV A2 infected cell culture media (at $48 \mathrm{~h}$ ) compared with control mock-noninfected media. Each lane represents a sample from a separate well. $50 \mu \mathrm{g}$ protein per sample was loaded into each well. 50 ng of recombinant MMP-9 (\#WBC018; R\&D Systems, Minneapolis, MN, USA) was used as a positive control. C: cell culture media obtained from mock-infected cells (with Hep-2 media); R: media obtained from RSV-infected HAECs (multiplicity of infection (MOI) 0.1); rh: recombinant human; A: "apical" cell culture media, obtained from the apical compartment of RSV-infected monolayers (MOI 0.1); B: "basolateral" media, obtained from the basolateral compartment.

\section{Results}

RSV infection induces robust MMP-9 expression in primary HAECs

MMP-9 mRNA levels were increased in primary HAECs infected with RSV A2 strain compared with controls following $48 \mathrm{~h}$ of infection (fig. 1a). MMP-9 gene expression was specific to RSV infection, as exposure to killed virus (heat killed or UV light treated) and adenovirus failed to stimulate increases in MMP-9 mRNA. Fig. $1 b$ shows that MMP-9 mRNA increased steadily with increasing RSV MOI (0.05-10, 48-h time point) and with duration of infection (MOI 0.1) (fig. 1c). In contrast to MMP-9, TIMP-1 mRNA levels were similar between RSV-infected and noninfected airway epithelial cells on day 2 and 6 postinfection (fig. 1d).

We next examined MMP-9 protein release in HAECs post-RSV infection. Figure 2 demonstrates extracellular release of MMP-9 protein after RSV infection (48 h and MOI 0.1), with greater release observed in the apical compartment relative to the basolateral compartment. MMP-9 protein concentrations correlated with the increased in MMP-9 mRNA seen on day 2 post-infection (fig. 1). In contrast to MMP-9, TIMP-1 protein was undetectable in the RSV-infected cell media (data not shown). Transepithelial resistance (TER) was monitored as a surrogate for monolayer integrity, and in the RSVinfected cells, TER did not decrease from the time of infection (mean \pm SEM $453 \pm 62 \Omega \cdot \mathrm{cm}^{-2}$ ) through day 4 post-infection (mean \pm SEM $722 \pm 19 \Omega \cdot \mathrm{cm}^{-2}$ ).

MMP-9 activity and total MMP-9 levels are elevated in infants with RSV-RF compared to controls Zymography was initially used to estimate MMP-9 activity in the endotracheal aspirates obtained from control and RSV-RF subjects. At $48 \mathrm{~h}$ of intubation, endogenous MMP-9 levels were present in lung secretions of RSV-RF subjects and noninfected controls, with more robust activity seen in infants with RSVRF (fig. 3a). Significantly higher amounts of active MMP-9 were measured in subjects with RSV-RF when compared with both control groups (using ELISA) (fig. 3b). Increases in active MMP-9 seen in the RSV-RF cohort remained significant $(\mathrm{p}<0.001)$ after adjustment for differences in age, sex, disease severity (oxygen requirement and PRISM scores) and other clinical outcome measures (duration of intubation and paediatric ICU stay) between the RSV-RF and control subjects by multivariate analysis.

To assess the total MMP-9 levels in tracheal aspirates, aminophenylmercuric acetate (APMA), a known chemical activator of pro-MMP-9 was added to selected samples. Complementary samples without the addition of APMA measured the amount of active MMP-9 present in the samples (fig. 3b). The highest total MMP-9 levels were observed in the RSV-RF subjects compared with the control groups, and this increase in total MMP-9 concentration (including both basal activity and inducible pro-enzyme) remained statistically significant after adjustment for age, sex, disease severity and outcome measures $(\mathrm{p}<0.001)$.

TIMP-1 concentrations are decreased in RSV-RF leading to elevated MMP-9/TIMP-1 activity ratios TIMP-1 serves as an endogenous inhibitor of MMP-9 that binds to MMP-9 in a 1:1 stoichiometric ratio [7]. The lowest concentration of TIMP-1 was measured in tracheal aspirates from RSV-RF subjects compared with controls (fig. 3c), contributing to significantly higher MMP-9/TIMP-1 activity ratios seen in RSV-RF subjects (fig. 3d). Following multivariate analysis to adjust for differences in age, sex and disease severity and outcome between RSV-RF, non-RSV pneumonia and nonlung disease controls, the elevated MMP-9/ TIMP-1 ratios seen in the RSV-RF cohort remained significant $(\mathrm{p}<0.001)$ at the 48 -h time point.

\section{Elevated MPO and HNE levels in RSV-RF}

As surrogates for neutrophil influx into the lung, MPO and HNE concentrations were measured in endotracheal lavage samples of RSV and control subjects. At $48 \mathrm{~h}$ of mechanical ventilation, RSV-RF 
a)
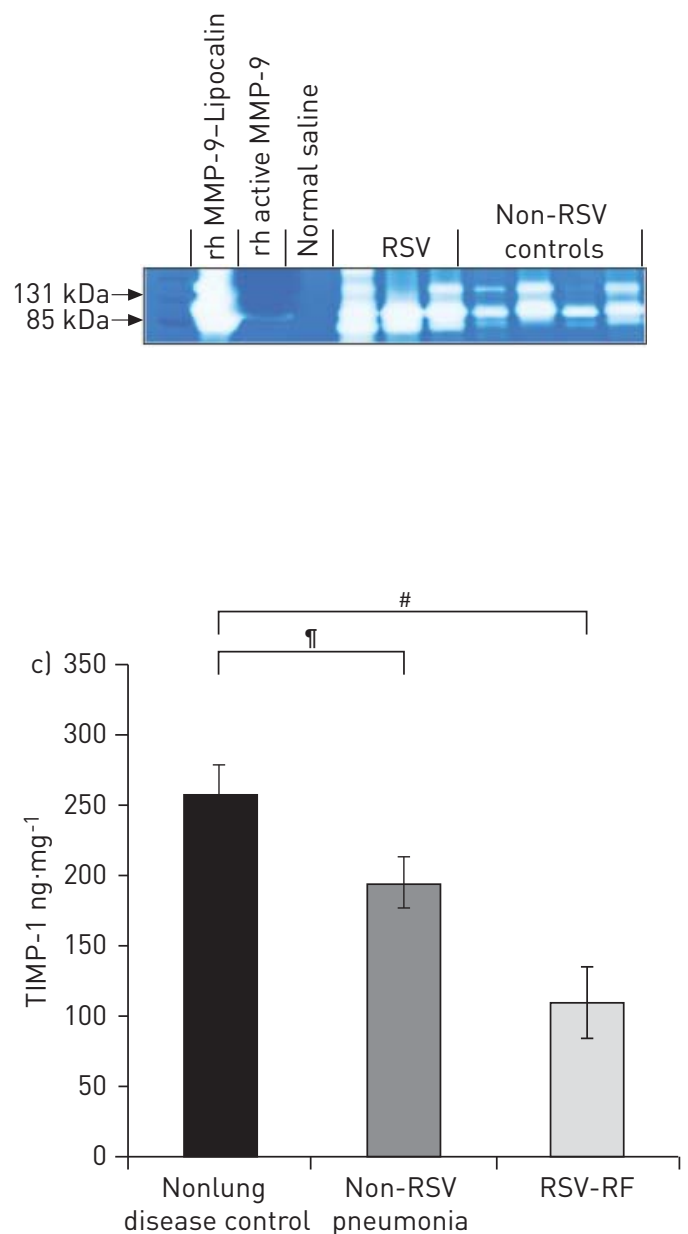
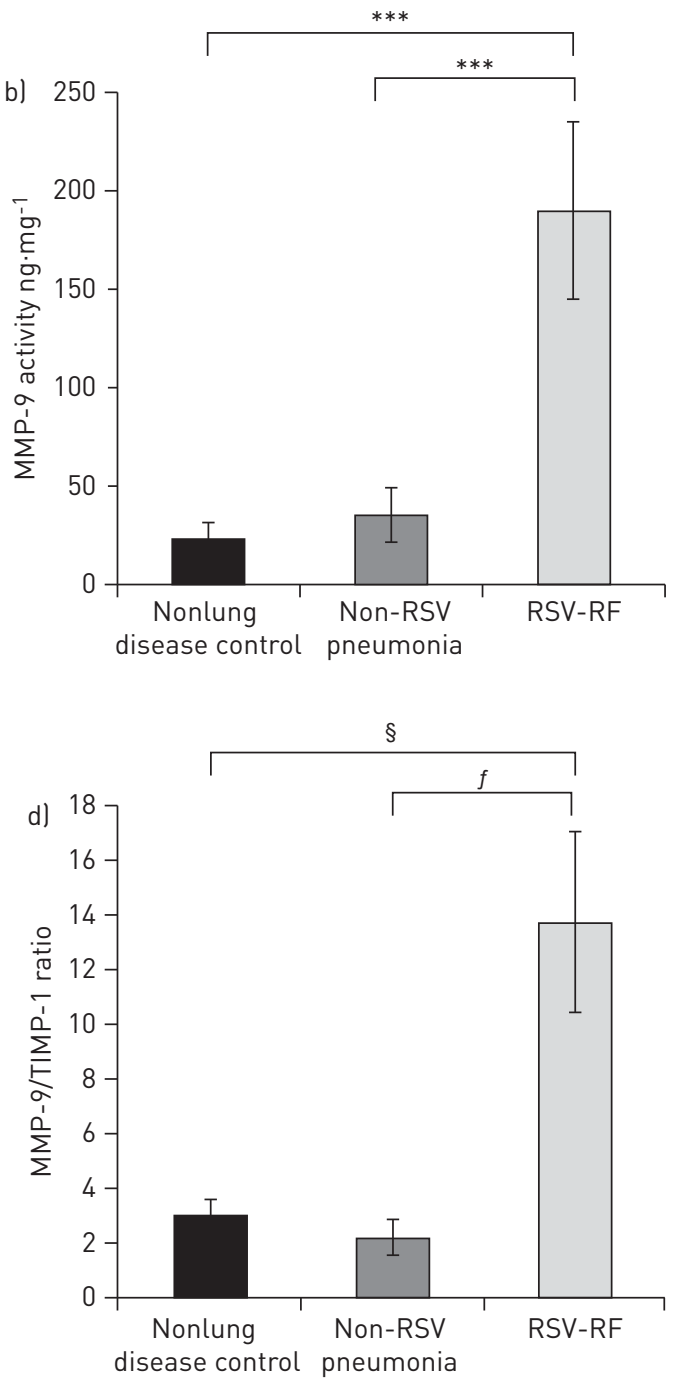

FIGURE 3 Dysregulated matrix metalloproteinase (MMP)-9 activity was observed in mechanically ventilated infants with respiratory syncytial virus (RSV)-induced respiratory failure (RF). a) Zymogram (7.5\% SDS nonreduced gel) demonstrates robust gelatinase activity in tracheal aspirates from RSV-RF subjects. Each lane represents a sample obtained from separate RSV-RF and control noninfected subjects at $48 \mathrm{~h}$ of mechanical ventilation and onset of RF. In brief, samples $(8 \mu \mathrm{g}$ protein per sample) were subjected to electrophoresis through $7.5 \%$ polyacrylamide gels containing $1 \mathrm{mg} \cdot \mathrm{mL}^{-1}$ porcine skin gelatine in the presence of SDS under nonreducing conditions. Lytic areas within the gel represent MMP-9 activity present in samples. The higher molecular weight band at $135 \mathrm{kDa}$ represents lipocalin-MMP-9 complexes, while the 82-kDa band corresponds with active MMP-9 isoforms. $25 \mathrm{ng}$ of recombinant lipocalin-MMP-9 complexes (\#444233; R\&D Systems, Minneapolis, MN, USA) and $20 \mathrm{ng}$ of recombinant active MMP-9 (\#PF024-5UG; Millipore, Billerica, MA, USA) were used as positive controls. Normal saline (utilised for sample processing) was used as negative control. b) Concentrations of endogenously active MMP-9 (i.e. prior to aminophenylmercuric acetate stimulation) were approximately five- and nine-fold higher in tracheal aspirates from patients with RSV-RF $(n=42)$ when compared to non-RSV pneumonia $(n=29)$ and nonlung disease controls $(n=46)$ (mean $\pm \mathrm{sEM} 90 \pm 44 \mathrm{ng} \cdot \mathrm{mg}^{-1}$ versus $35 \pm 14 \mathrm{ng} \cdot \mathrm{mg}^{-1}$ and $22.9 \pm 8.1 \mathrm{ng} \cdot \mathrm{mg}^{-1}$, respectively). c) The lowest concentrations of tissue inhibitor of metalloproteinase (TIMP)-1 were measured in the tracheal aspirates from RSV-RF subjects compared with non-RSV pneumonia and nonlung disease control groups. TIMP-1 level in RSV-RF subjects was approximately two-fold lower than in the nonlung disease controls (mean \pm SEM $110 \pm 24 \mathrm{ng} \mathrm{mg}^{-1}$ versus $257 \pm 49 \mathrm{ng} \cdot \mathrm{mg}^{-1}$ ). The amount of measured TIMP-1 in the non-RSV pneumonia group (mean \pm SEM $194 \pm 58 \mathrm{ng} \cdot \mathrm{mg}^{-1}$ ) was also lower than the nonlung disease controls. d) Highest MMP-9/TIMP-1 activity ratios were observed in RSV-RF subjects compared to non-RSV pneumonia and nonlung disease controls (mean \pm SEM $13.7 \pm 2.9$ versus $2.1 \pm 0.6$ and $2.9 \pm 0.6$, respectively), probably secondary to a combination of higher MMP-9 activity and depressed TIMP-1 levels found in the RSV-RF subjects (b and $c$ ). rh: recombinant human. ${ }^{* * *}: p<0.001 ;{ }^{*}: p=0.006$; ๑: $\mathrm{p}=0.022 ;{ }^{\S}: \mathrm{p}=0.001 ;{ }^{\dagger}: \mathrm{p}=0.005$. 

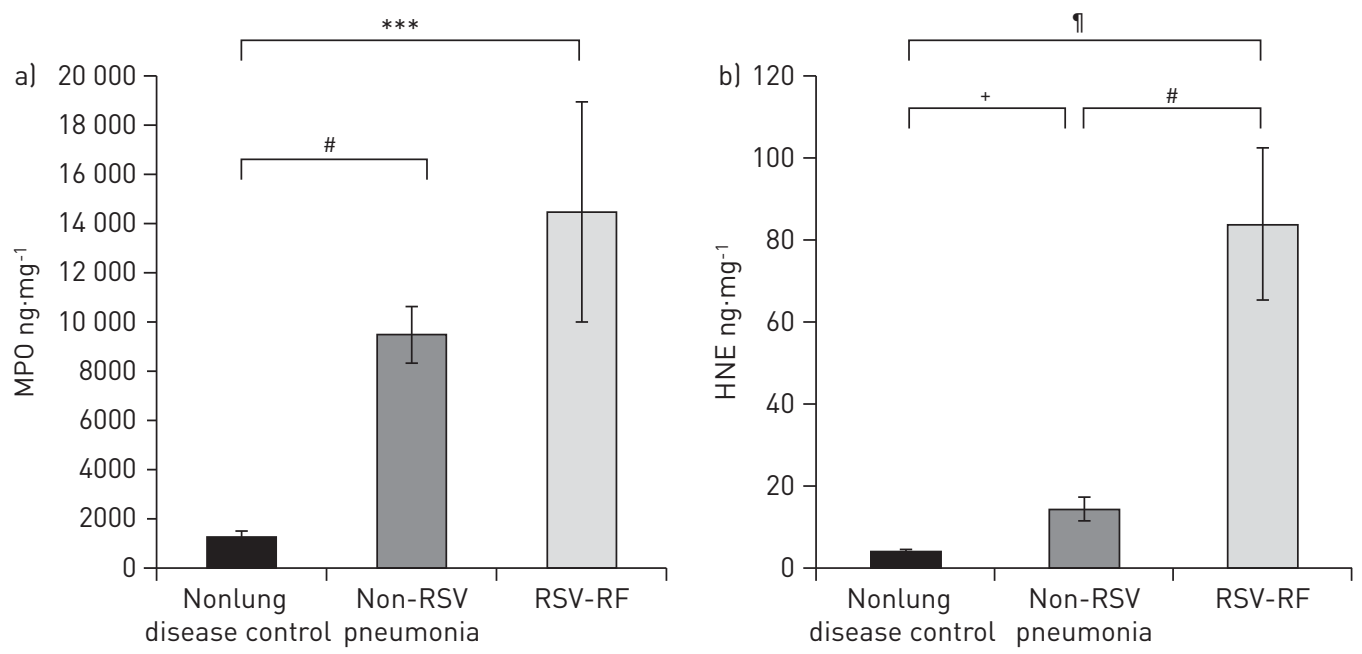

FIGURE 4 The highest myeloperoxidase (MPO) and human neutrophil elastase (HNE) levels were found in respiratory syncytial virus (RSV)-induced respiratory failure (RF) subjects. a) MPO levels from RSV-RF $(n=42)$ and non-RSV pneumonia $(n=29)$ patients were found to be approximately 12 -fold and seven-fold higher than in nonlung disease controls ( $\mathrm{n}=46)$ (mean \pm SEM $14469 \pm 4436 \mathrm{ng} \cdot \mathrm{mg}^{-1}$ versus $9486 \pm 1154 \mathrm{ng} \cdot \mathrm{mg}^{-1}$ versus $1208 \pm 278 \mathrm{ng} \cdot \mathrm{mg}^{-1}$, respectively). b) HNE levels were approximately 20 -fold and 3.5 -fold higher in the RSV-RF cohort and non-RSV pneumonia subjects compared to the nonlung disease controls (mean \pm SEM $84 \pm 18 \mathrm{ng} \cdot \mathrm{mg}^{-1}$ versus $14 \pm 2.9 \mathrm{ng} \cdot \mathrm{mg}^{-1}$ versus $4.2 \pm 0.6 \mathrm{ng} \cdot \mathrm{mg}^{-1}$, respectively). The highest level of HNE was measured in the RSV-RF cohort, with an approximate six-fold increase seen in this cohort compared to the non-RSV pneumonia controls. ${ }^{* * *}: \mathrm{p}<0.001 ;^{*}: \mathrm{p}=0.001 ;{ }^{\natural}: \mathrm{p}<0.0001 ;{ }^{+}: \mathrm{p}=0.0001$.

patients had the highest levels of MPO and HNE of the three groups (fig. 4). In the RSV cohort, endotracheal MPO and HNE levels demonstrated a positive correlation $(r=0.4, p=0.017)$, while no correlation was observed between MMP-9 concentration with either HNE or MPO.

\section{MMP-9/TIMP-1 ratios remained elevated with disease progression in RSV-RF}

Next, we analysed MMP-9 activity and MMP-9/TIMP-1 ratios in the subgroup of RSV-RF subjects and controls who required $>48 \mathrm{~h}$ of ventilatory support. In the RSV-RF subjects $(\mathrm{n}=8)$, MMP-9 activity remained high with disease progression (mean \pm SEM at $48 \mathrm{~h} \quad 170 \pm 90 \mathrm{ng} \cdot \mathrm{mg}^{-1}$ versus day 5 $\left.399 \pm 47 \mathrm{ng} \cdot \mathrm{mg}^{-1} ; \mathrm{p}=0.05\right)$. TIMP-1 levels were decreased at day 5 of mechanical ventilation, leading to

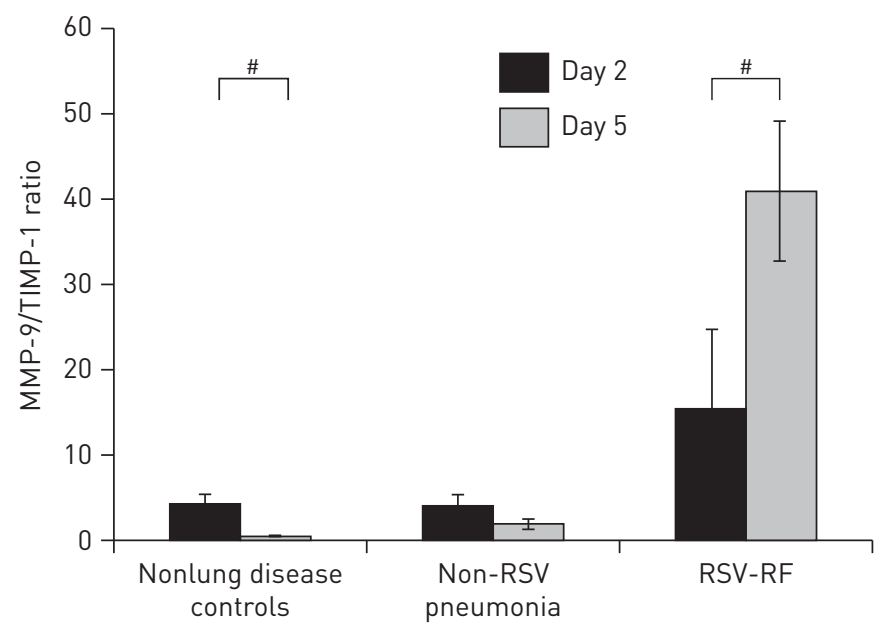

FIGURE 5 Matrix metalloproteinase (MMP)-9/tissue inhibitor of metalloproteinase (TIMP)-1 ratios remained elevated with time in respiratory syncytial virus (RSV)-induced respiratory failure (RF) subjects versus resolution in control groups. Persistently elevated MMP-9/TIMP-1 ratios were seen with time in the RSV-RF subjects (n=8) during mechanical ventilation (mean + SEM at $48 \mathrm{~h} 15+8$ versus day $541+8$ ). In contrast to RSV-RF subjects, nonlung disease controls $(n=9)$ demonstrated lower MMP-9/TIMP-1 ratios in their tracheal aspirates by day 5 of mechanical ventilation (mean \pm SEM at $48 \mathrm{~h} 4 \pm 0.1$ versus day $50.2 \pm 0.05)$. Similarly, subjects with non-RSV pneumonia $(\mathrm{n}=13$ ) had a trend towards decreased MMP-9/TIMP-1 ratios (mean \pm SEM at $48 \mathrm{~h} 3.8 \pm 1 \mathrm{ng} \cdot \mathrm{mg}^{-1}$ versus day $51.8 \pm 0.5$; nonsignificant) by day 5 of intubation. ${ }^{*}: \mathrm{p}=0.04$. 
persistently elevated MMP-9/TIMP-1 ratios in the RSV-RF subjects (fig. 5). In contrast, MMP-9/TIMP-1 ratios were decreased with time in both the non-RSV pneumonia $(n=13)$ and nonlung-disease control groups $(n=9)$.

\section{Higher MMP-9 activity at $48 \mathrm{~h}$ correlated with higher oxygen requirement and worse PRISM scores in RSV-RF subjects}

At $48 \mathrm{~h}$ of intubation, active MMP-9 levels were positively correlated $(\mathrm{r}=0.74, \mathrm{p}<0.001)$ with oxygen requirement in the RSV-RF subjects, which was confirmed with multiple regression analyses, after adjusting for age, sex and disease severity (e.g. PRISM scores) and other clinical outcome measures (length of intubation and paediatric ICU stay) (table 2). A positive correlation was also seen between total MMP-9 with PRISM-12 scores at $48 \mathrm{~h}$ of disease onset $(\mathrm{r}=0.41, \mathrm{p}=0.022)$ in the RSV-RF subjects, which was confirmed by multiple linear regression analyses after controlling for age, sex, disease severity and outcome measures (table 2). In contrast to MMP-9 activity, neither MPO nor HNE levels demonstrated significant correlation with oxygen requirement, PRISM scores, duration of mechanical ventilation or paediatric ICU stay in the RSV cohort. In the control groups, no clear correlation was observed between the measured biomarkers (active MMP-9, total MMP-9, TIMP-1, MMP-9/TIMP-1 ratio, HNE and MPO) and clinical markers of disease severity at $48 \mathrm{~h}$ (oxygen requirement and PRISM scores), or subsequent clinical outcome measures (duration of mechanical ventilation and length of paediatric ICU stay).

\section{Discussion}

Our results highlight a unique mechanism by which RSV initiates MMP-9 dysregulation, stimulating MMP-9 transcription and MMP-9 protein release from infected human airway epithelial cells (independent of other host cell types). MMP-9 release occurs before loss of airway epithelial monolayer integrity, suggesting that RSV-stimulation of MMP-9 production and release from HAECs is an early step in RSV disease pathogenesis prior to overt epithelial toxicity. In patients with RSV infection, we found elevated levels of active MMP-9 compared to a control population and an imbalance of MMP-9/TIMP-1 ratio in RSV-infected patients at $48 \mathrm{~h}$ of intubation. MMP-9 activity and MMP-9/TIMP-1 ratios continued to increase over the course of mechanical ventilation in the RSV cohort, in contrast to depressed levels seen in the non-RSV pneumonia and nonlung disease control groups. Thus, our data supports the notion that endotracheal MMP-9 is a specific marker for RSV disease severity in mechanically ventilated infants with RSV-RF.

Few studies have examined the relationship between RSV infection and MMP-9 induction in the lung. YEO et al. [25] reported increased MMP-9 release from HEp-2 cells with RSV stimulation at 24 h post-infection. In animal models, RSV infection caused an increase in MMP-9 transcription and protein release in bronchoalveolar lavage fluid [26]. In a more recent study, RSV infection in an immortalised human bronchial epithelial cell line caused upregulation of MMP-9 transcription, but MMP-9 protein was not detected in the cell culture supernatants [27]. The authors concluded that airway epithelial cells were not the primary source of MMP-9 following a paramyxovirus infection, and that the increase in MMP-9 protein release observed in the A549 cells and HEp-2 cells was due, in part, to the tumorogenic properties of the cell lines. In contrast to these studies, we found significant elevation of MMP-9 transcription and protein release in the RSV-infected primary HAECs when compared with control noninfected cells. The use of primary HAECs in our studies may provide a more physiological model for studies of MMP-9 and human RSV infection relative to studies in immortalised cell lines and in animal models. In this model, the airway cells demonstrate a pseudostratified, polarised phenotype, including ciliated and goblet cells, and tight monolayer integrity as measured by high TER. Control experiments utilising UV-inactivated RSV (which

TABLE 2 Positive correlation between measured biomarkers in the respiratory syncytial virus (RSV) cohort and clinical measures by multiple linear regression

PRISM-12

PRISM-24

Oxygen concentration

$\begin{array}{cc}\text { NS } & 0.57(p<0.001) \\ \text { NS } & 0.37(p<0.001) \\ \text { NS } & 0.35(p=0.002) \\ \text { NS } & \text { NS } \\ \text { NS } & \text { NS }\end{array}$

\section{Length of intubation}

$\begin{array}{ll}\text { NS } & \text { NS } \\ \text { NS } & \text { NS } \\ \text { NS } & \text { NS } \\ \text { NS } & \text { NS } \\ \text { NS } & \text { NS }\end{array}$

Data are presented as statistically significant $r^{2}$ values, unless otherwise stated. PRISM-12: paediatric risk of mortality in $12 \mathrm{~h}$; PRISM-24: paediatric risk of mortality in $24 \mathrm{~h}$; PICU: paediatric intensive care unit; MMP: matrix metalloproteinase; TIMP: tissue inhibitor of metalloproteinase; MPO: myeloperoxidase; HNE: human neutrophil elastase; NS: not significant. 
eliminates viral infectivity without altering the conformation of viral proteins) and heat-killed RSV (which eliminates viral infectivity and changes protein structures) failed to stimulate MMP-9 expression, indicating the need for live replicating RSV virus to induce MMP-9 production. Furthermore, MMP-9 mRNA levels increased steadily with increasing MOI but reached a plateau at MOI $=1$, supporting the notion that viral replication (versus structural viral proteins alone) were key in stimulating MMP-9 production.

A variety of other cells produce MMP-9 in the lung including tissue macrophages, sequestered neutrophils and fibroblasts [28]. In addition to epithelial cells, it has been shown that TIMP-1 can be produced from both interstitial and alveolar macrophages. Therefore, although our study highlights the impact of RSV on airway epithelial release and regulation of MMP-9, it is possible that RSV also impacts the regulation and release of these proteins from other cell types in the lung.

Although our cellular findings demonstrate the transcription of MMP-9 and release of pro-MMP-9 following RSV infection, our human data suggest that a large proportion of the detectable MMP-9 was in the active form. RSV-RF subjects also had lower TIMP-1 levels, resulting in higher MMP-9/TIMP-1 ratios during acute disease and with time on the ventilator. Our group has recently demonstrated that HNE is capable of converting pro-MMP-9 to active enzyme, and can independently degrade TIMP-1 [14]. Here, we found high levels of HNE in the RSV lung samples compared to controls (approximately six-fold and 21-fold higher than non-RSV pneumonia subjects and nonlung disease controls, respectively). Thus, we speculate that HNE may be, in part, responsible for the high levels of active MMP-9 and low TIMP-1 levels observed in our RSV subjects. Furthermore, our in vitro studies demonstrated absent TIMP-1 protein release following RSV infection of HAECs, initiating conditions that predict high MMP-9/TIMP-1 ratios in vivo.

An imbalance between MMP-9 and TIMP-1 levels has been shown to correlate with airflow obstruction, diminished lung function and duration of ventilatory support [13-15, 29]. Furthermore, excessive MMP-9 activity has also been shown to contribute to increased mortality in mice following Francisella tularensis infection, as MMP-9 knockout mice demonstrated improved survival relative to MMP- $9^{+/+}$littermates with respiratory tularaemia [30]. To our knowledge, this is the first study to describe an association between high levels of active MMP-9, total MMP-9 and MMP-9/TIMP-1 ratios with disease severity in mechanically ventilated children with RSV-RF. Although HNE and MPO (markers of polymorphonuclear (PMN) infiltration) were elevated in the RSV-RF subjects, no correlation was found between these neutrophilderived enzymes and disease severity. A recent study demonstrated a link between disease severity of viral lower respiratory tract infections with increased expression of MMP-8 and MMP-9 genes in mononuclear cells and granulocytes [31]. However, no differences were noted in total MMP-9 concentrations in nasopharyngeal washes in children with severe disease compared with those with mild or moderate disease, and, in addition, MMP-9/TIMP-1 ratios were not increased during acute RSV infection. In that study, mild disease was defined as RSV without hypoxia, while moderate and severe disease were defined as need for oxygen and mechanical ventilation, respectively. Since only total MMP-9 concentration was measured, it remains unclear if active MMP-9 was different between those with mild, moderate or severe RSV disease. Another study demonstrated that nasopharyngeal samples from infants with RSV bronchiolitis contained increased MMP-9 and TIMP-1 concentrations, and that TIMP-1 correlated negatively with oxygen saturations [27]. In contrast, we observed that TIMP-1 alone in lower airway samples had no correlation with disease severity or clinical outcome measures. It is important to note that the study populations [27, 31] were different from our RSV cohort, in that all of our study subjects had respiratory failure necessitating ventilatory support. Furthermore, our studies focused only on lower airway samples from RSV-infected and control subjects, thus examining relationships between RSV and MMP-9 in the target organ of disease.

One potential limitation to our study is the confounding effect of mechanical ventilation, and the previous reports of elevated MMP-9 expression seen in patients with ventilator-induced lung injury [32]. In our study, all controls and study subjects received mechanical ventilation, thus controlling for the effect of mechanical ventilation on MMP-9 activity. Furthermore, high levels of MMP-9 have also been reported in other PMN-dominated inflammatory lung conditions, such as pneumonia and acute respiratory distress syndrome $[15,33]$. In this study, consistent with these previous reports, MMP-9, HNE and MPO activity were elevated in the non-RSV pneumonia subjects compared to nonlung injury controls. However, in our experience, the highest levels of MMP-9 activity were observed in the RSV-infected patients, and MMP-9 (active MMP-9 in particular) outperformed MPO and HNE as a predictor of disease severity. Another potential limitation to the findings of this study is the differences in age across study groups. Despite the varied patient demographics, we confirmed increased levels of MMP-9 activity in the RSV-RF subjects compared to the control groups by using multiple regression analysis; adjusting for differences in age, sex and clinical disease severity measures. We acknowledge that the relatively small number of control subjects with respiratory failure secondary to other viruses precluded a direct comparison between RSV and other viral pathogens. Others have shown correlation between high RSV titres and disease severity, suggesting that 
viral burden is the driving force behind the inflammation and deranged pathology observed in RSV infection $[34,35]$. In our studies, viral load was not quantified in the lung samples, therefore preventing direct analysis of RSV titres and the measured inflammatory markers with clinical outcome measures. Finally, due to the age and severity of disease of our patient population, bronchoalveolar lavages were not consistently performed. We instead used endotracheal aspirates as the safest method for sample collection in our studies. It is possible that cellularity and enzymatic profile in the alveolar compartment is different relative to that seen in tracheal-derived secretions. In our studies, we employed standardised sampling techniques and adjusted the MMP, TIMP, HNE and MPO levels for the amount of protein present in the sample. The use of endotracheal samples may also have practical implications, as endotracheal aspirates are simple to obtain and thus may improve the portability of our findings.

Together, our findings suggest that dysregulation of MMP-9 activity and disruption of MMP-9/TIMP-1 homeostasis are key factors contributing to inflammation and clinical disease manifestations in the RSVinfected lung. Our in vitro results in isolated airway epithelial cell monolayers identified RSV infection as a specific stimulus of MMP-9 expression and release independent of nonepithelial cells, and thus provides a rationale for close correlations between MMP-9 and clinical markers of disease (as opposed to other markers of neutrophil influx). These findings are novel and justify future validation studies in larger patient cohorts, especially to examine MMP-9 imbalance with other previously reported markers of disease severity in RSV infection, such as viral burden, interleukin (IL)-4 and IL-8 gene polymorphisms and MMP-3 [34-37], as this may provide greater prognostic values than each marker individually.

\section{Conclusion}

Our studies identify a direct link between RSV infection and MMP-9 transcription and protein release in human airway epithelial cells. Dysregulated MMP-9 activity, as demonstrated by high levels of active MMP-9 and MMP-9/TIMP-1 imbalance at 48 h of intubation, correlated with higher oxygen requirement and PRISM scores in mechanically ventilated children with RSV-RF in this single-centre study. Together, the findings support investigation of RSV and MMP-9 as a mediator of RSV-dependent tissue injury, potentially identifying MMP-9 as a novel biomarker and therapeutic target for RSV disease.

\section{Acknowledgements}

The authors thank the paediatric intensive care unit nurses and respiratory therapists (Children's of Alabama, Birmingham, AL, USA) for their assistance with tracheal aspirate collections. They also thank the Cystic Fibrosis Clinical and Translational Core at the Gregory Fleming James Cystic Fibrosis Research Center of University of Alabama at Birmingham for providing us with primary human airway epithelial cells used in the in vitro experiments. Finally, the authors thank Eric Sorscher (University of Alabama at Birmingham, Birmingham, AL, USA) for his helpful discussion of the manuscript.

\section{References}

Hacking D, Hull J. Respiratory syncytial virus - viral biology and the host response. J Infect 2002; 45: 18-24. Purcell K, Fergie J. Driscoll Children's Hospital respiratory syncytial virus database: risk factors, treatment and hospital course in 3308 infants and young children, 1991 to 2002. Pediatr Infect Dis J 2004; 23: 418-423.

3 Chmiel JF, Davis PB. State of the art: why do the lungs of patients with cystic fibrosis become infected and why can't they clear the infection? Respir Res 2003; 4: 8.

4 Ramaswamy M, Groskreutz DJ, Look DC. Recognizing the importance of respiratory syncytial virus in chronic obstructive pulmonary disease. COPD 2009; 6: 64-75.

5 Stein RT, Sherrill D, Morgan WJ, et al. Respiratory syncytial virus in early life and risk of wheeze and allergy by age 13 years. Lancet 1999; 354: 541-545.

6 Elkington PT, Friedland JS. Matrix metalloproteinase in destructive pulmonary pathology. Thorax 2006; 61: 259-266.

7 Birkedal-Hansen H, Moore WG, Bodden MK, et al. Matrix metalloproteinases: a review. Crit Rev Oral Biol Med 1993; 4: 197-250.

8 Tanaka H, Miyazaki N, Oashi K, et al. Sputum matrix metalloproteinase-9: tissue inhibitor of metalloproteinase-1 ratio in acute asthma. J Allergy Clin Immunol 2000; 105: 900-905.

9 Cataldo DD, Bettiol J, Noël A, et al. Matrix metalloproteinase-9, but not tissue inhibitor of matrix metalloproteinase-1, increases in the sputum from allergic asthmatic patients after allergen challenge. Chest 2002; 122: 1553-1559.

10 Betsuyaku T, Nishimura M, Takeyabu K, et al. Neutrophil granule proteins in bronchoalveolar lavage fluid from subjects with subclinical emphysema. Am J Respir Crit Care Med 1999; 159: 1985-1991.

11 Culpitt SV, Rogers DF, Traves SL, et al. Sputum matrix metalloproteinases: comparison between chronic obstructive pulmonary disease and asthma. Respir Med 2005; 99: 703-710.

12 Cederqvist K, Sorsa T, Tervahartiala T, et al. Matrix metalloproteinases-2, -8 , and -9 and TIMP-2 in tracheal aspirates from preterm infants with respiratory distress. Pediatrics 2001; 108: 686-692.

13 Sagel SD, Kapsner RK, Osberg I. Induced sputum matrix metalloproteinase-9 correlates with lung function and airway inflammation in children with cystic fibrosis. Pediatr Pulmonol 2005; 39: 224-232.

14 Gaggar A, Li Y, Weathington N, et al. Matrix metalloprotease-9 dysregulation in lower airway secretions of cystic fibrosis patients. Am J Physiol Lung Cell Mol Physiol 2007; 293: L96-L104. 
Kong M, Gaggar A, Li Y, et al. Matrix metalloproteinase activity in pediatric acute lung injury. Int J Med Sci 2009; 6: 9-17.

16 Weathington N, van Houwelingen A, Noerager B, et al. A novel peptide CXCR ligand derived from extracellular matrix degradation during airway inflammation. Nat Med 2006; 12: 317-323.

$17 \mathrm{Xu} \mathrm{X}$, Jackson PL, Tanner S, et al. A self-propagating matrix metalloprotease-9 (MMP-9) dependent cycle of chronic neutrophilic inflammation. PLoS One 2011; 6: e15781.

18 Kong M, Li Y, Oster R, et al. Early elevation of matrix metalloproteinase- 8 and -9 in pediatric ARDS is associated with an increased risk of prolonged mechanical ventilation. PLoS One 2011; 6: e22596.

19 Sloane PA, Shastry S, Wilhelm A, et al. A pharmacologic approach to acquired cystic fibrosis transmembrane conductance regulator dysfunction in smoking related lung disease. PLoS One 2012; 7: e39809.

20 Mbiguino A, Menezes J. Purification of human respiratory syncytial virus: superiority of sucrose gradient over percoll, renografin, and metrizamide gradients. J Virol Methods 1991; 31: 161-170.

21 Sullender WM, Anderson K, Wertz GW. The respiratory syncytial virus subgroup B attachment glycoprotein: analysis of sequence, expression from a recombinant vector, and evaluation as an immunogen against homologous and heterologous subgroup virus challenge. Virology 1990; 178: 195-203.

22 Jaovisidha P, Peeples ME, Brees AA, et al. Respiratory syncytial virus stimulates neutrophil degranulation and chemokine release. J Immunol 1999; 163: 2816-2820.

23 Hibbs MS, Hasty KA, Seyer JM, et al. Biochemical and immunological characterization of the secreted forms of human neutrophil gelatinase. J Biol Chem 1985; 260: 2493-2500.

24 Laemmli UK. Cleavage of structural proteins during the assembly of the head of bacteriophage T4. Nature 1970; 227: 680-685.

25 Yeo SJ, Yun YJ, Lyu MA, et al. Respiratory syncytial virus infection induces matrix metalloproteinase-9 expression in epithelial cells. Arch Virol 2002; 147: 229-242.

$26 \mathrm{Li} \mathrm{W}$, Shen HH. Effect of respiratory syncytial virus on the activity of matrix metalloproteinase in mice. Chin Med J (Engl) 2007; 120: 5-11.

27 Elliott MB, Welliver RC Sr, Laughlin TS, et al. Matrix metalloproteinase-9 and tissue inhibitor of matrix metalloproteinase-1 in the respiratory tracts of human infants following paramyxovirus infection. J Med Virol 2007; 79: 447-456. Atkinson JJ, Senior RM. Matrix metalloproteinase-9 in lung remodeling. Am J Respir Cell Mol Biol 2003; 28: 12-24. Matsumoto H, Niimi A, Takemura M, et al. Relationship of airway wall thickening to an imbalance between matrix metalloproteinase-9 and its inhibitor in asthma. Thorax 2005; 60: 277-281.

30 Malik M, Bakshi CS, McCabe K, et al. Matrix metalloproteinase 9 activity enhances host susceptibility to pulmonary infection with type A and B strains of Francisella tularensis. J Immunol 2007; 178: 1013-1020.

31 Brand KH, Ahout IM, de Groot R, et al. Use of MMP-8 and MMP-9 to assess disease severity in children with viral lower respiratory tract infections. J Med Virol 2012; 84: 1471-1480.

32 González-López A, Astudillo A, García-Prieto E, et al. Inflammation and matrix remodeling during repair of ventilator-induced lung injury. Am J Physiol Lung Cell Mol Physiol 2011; 301: L500-L509.

33 Wilkinson TS, Morris AC, Kefala K, et al. Ventilator-associated pneumonia is characterized by excessive release of neutrophil proteases in the lung. Chest 2012; 142: 1425-1432.

34 El Saleeby CM, Bush AJ, Harrison LM, et al. Respiratory syncytial virus load, viral dynamics, and disease severity in previously healthy naturally infected children. J Infect Dis 2011; 204: 996-1002.

35 DeVincenzo JP, Wilkinson T, Vaishnaw A, et al. Viral load drives disease in humans experimentally infected with respiratory syncytial virus. Am J Respir Crit Care Med 2010; 182: 1305-1314.

36 Hoebee B, Rietveld E, Bont L, et al. Association of severe respiratory syncytial virus bronchiolitis with interleukin-4 and interleukin-4 receptor $\alpha$ polymorphisms. J Infect Dis 2003; 187: 2-11.

37 Schuurhof A, Bont L, Hodemaekers HM, et al. Proteins involved in extracellular matrix dynamics are associated with respiratory syncytial virus disease severity. Eur Respir J 2012; 39: 1475-1481. 